\title{
Evaluation of Clinical Efficacy on Acute Pancreatitis Treated with Combination of Traditional Chinese and Western Medicine: A Meta-Analysis
}

\author{
Jixiang Ao, Hui Long* \\ Department of Gastroenterology, Tianyou Hospital, Wuhan University of Science and Technology, Wuhan, China \\ Email: *1605109246@qq.com
}

How to cite this paper: Ao, J.X. and Long, H. (2018) Evaluation of Clinical Efficacy on Acute Pancreatitis Treated with Combination of Traditional Chinese and Western Medicine: A Meta-Analysis. International Journal of Clinical Medicine, 9, 249-259. https://doi.org/10.4236/ijcm.2018.94022

Received: March 17, 2018

Accepted: April 17, 2018

Published: April 20, 2018

Copyright ( 92018 by authors and Scientific Research Publishing Inc. This work is licensed under the Creative Commons Attribution International License (CC BY 4.0).

http://creativecommons.org/licenses/by/4.0/ (c) (i) Open Access

\begin{abstract}
Objective: Meta-analysis was conducted to assess whether combination of traditional Chinese and western medicine could enhance clinical outcomes compared to single western medicine in the treatment of acute pancreatitis (AP). Methods: This systematic review will include prospective and retrospective comparative studies in all languages and evaluate the clinical efficacy of integrated traditional Chinese and western medicine versus single western medicine in treatment AP. Animal studies will not be considered. Published articles were acquired from Jan. 2001 to Dec. 2017 by a comprehensive search in Chinese National Knowledge Infrastructure database (CNKI), Chinese WANFANG database. Exclusion criteria were non-comparative studies, hybrid treatment. The main points include clinical efficacy; carefully evaluating and reviewing the literature and filtering the literature according to inclusion criteria and exclusion criteria. Statistical analyses were performed using software Review Manager (Version 5.0). Publication biases of main results were examined by Stata 12.0. Results: 1262 cases of 1342 cases were effective and the effective rate reaches $94.04 \%$ in the group of integrated traditional Chinese and western medicine, while 949 cases of 1223 cases were effective and its effective rate can be up to $77.60 \%$ in the group of single western medicine $(P>$ $0.05 \%, I^{2}=0 \%$, Odds Ratio $(O R)=4.98,95 \% \mathrm{CI}=3.79-6.54, P<0.01$.). Conclusion: These results suggest that the therapeutic effects of integrated traditional Chinese and Western medicine on AP are better in treatment of AP than that of western medicine alone.
\end{abstract}

\section{Keywords}

Combination of Traditional Chinese and Western Medicine, Acute 
Pancreatitis, Qingyi Decoction, Randomized Control

\section{Introduction}

Acute pancreatitis, as an inflammatory reaction, could activate pancreatic enzymes in the pancreas and lead to pancreatic tissue autodigestion, edema, hemorrhage and even necrosis. Clinical features of the disease are acute epigastric pain, nausea, vomit, fever and aberrant serum trypsin. Different period patients have different symptoms. The mild symptoms are mainly showed in pancreatic edema and owned to self-limited trait. While pancreatic hemorrhage necrosis, secondary infection, peritonitis and shock are common in severe acute pancreatitis. Therefore, acute pancreatitis was regarded as a critical and high mortality disease in the Department of Digestive Medicine. It caused to pay enough attention to this disease by clinicians. Meanwhile, international guidelines have recommended clearly the treatment of internal medicine for acute pancreatitis. At present, the routine diagnosis and treatment of western medicine has formed a mature treatment plan, such as fasting, PPI, somatostatin, ulinastatin, intravenous nutrition, and so on. Chinese clinicians and researchers found that the effective rate of the treatment was significantly improved after taking orally/decoction with Qingyi soup on the basis of western medicine treatment. Qingyi Decoction is a traditional Chinese medicine prescription composed of Radix Bupleurum, Radix Paeoniae Alba and Radix and Rhizoma et al., which has the effect of soothing the liver and regulating qi, clearing heat and detoxifying, and removing stasis by purgation. Meanwhile, many clinicians and researchers have explored relative merits in the treatment of acute pancreatitis with combination of traditional Chinese and western medicine and simple treatment of acute pancreatitis via randomized double-blind controlled experiments. The effective rate of combination of traditional Chinese and western medicine in treating acute pancreatitis was significantly superior to single western medicine, but acute pancreatitis was not frequent, so the sample size in most studies was not large enough to reflect the overall effective rate. The purpose of this paper is to make quantitative analysis and objective analysis in order to remove the batch effect and avoid the limitation of the conclusion of a single small sample. Then, we also conducted to increase sample number in order to increase the credibility of the literature and identify some unclear issues in individual studies. The general steps for meta-analysis include: Literature retrieval, Data extraction, Characteristic description, Quality evaluation of documents, Statistical analysis (1. Heterogeneity test; 2. Statistical merge effect; 3 . The results of single research-level data merging are shown by forest plot; 4 . The bias of published literature is understood by "funnel plot") and Conclusions and discussions, new research issues and prospects. Unfortunately, certain limitations still need to be considered while objective conditions cannot be incorporated into all relevant studies, so it cannot extract all the 
data. Furthermore, due to the influence of various factors included in the literature, publication bias still need to account.

\section{Materials and Methods}

\subsection{Inclusion Criteria}

1) The subjects of the study were patients with acute pancreatitis diagnosed in accordance with the Chinese diagnostic guidelines. This study did not subdivide mild, moderate and severe acute pancreatitis in patients with acute pancreatitis. 2) The effective rate of integrated traditional Chinese and western medicine therapy and that of western medicine alone was compared. This paper classifies "cure", "remarkable effect", "effective", "improvement" as "effective"; "invalid", "deterioration into surgery" and "death" as "ineffective". 3) Full text and raw data are available. 4) A randomized double-blind controlled trial was conducted. Exclude literature that meets one of the following conditions:

\subsection{Exclusion Criteria}

1) No control trial. 2) Unable to obtain raw data for the paper. 3) Republished literature. 4) Less than 30 cases per group. 5) The study published only abstract. 6) The study was not completed.

\subsection{Data Collection Methods}

Search CNKI database, WANFANG Data published on the treatment of acute pancreatitis with integrated Chinese and Western medicine articles, Chinese search keywords: "Qingyi Decoction; Combined treatment of traditional Chinese medicine and western medicine; Acute pancreatitis;" The English search keywords are "Qingyi Decoction, combined treatment of traditional Chinese medicine and western medicine, acute pancreatitis, RCT, meta-analysis".

\subsection{Data Extraction}

The following information was extracted from each study according to the above inclusion criteria and exclusion criteria. The data about the treatment of acute pancreatitis, such as "cure", "remarkable effect", "effective", "improvement" was seen as "effective"; "ineffective", "deterioration to surgical operation", "death" was seen as "ineffective". Select included standard article, and mark the first author and the year of the article published.

\subsection{Study Endpoint}

The effective rate of treatment was analyzed by Review Manager 5.0 software. The effective rates of each combination of traditional Chinese and western medicine in treating acute pancreatitis and that of western medicine alone in treating acute pancreatitis were calculated by relative risk. If $\mathrm{I}^{2}$ index was less than $50 \%$, it was regarded as no obvious heterogeneity among the results of these studies. The fixed effect model is used to analyze and process the data synthetically; the $95 \%$ 
$\mathrm{CI}$ and the probability value are calculated. If $\mathrm{I}^{2}$ index was more than $50 \%$, it was considered that there is obvious heterogeneity among the various studies. The data are analyzed synthetically and the random effect model is used to calculate the data. Finally, the forest plot and funnel plot are displayed.

\subsection{Statistical Processing}

Review Manager 5.0 software was used for statistical processing, and chi-square test was used to test the heterogeneity of the selected literature. The bilateral $P$ index was less than 0.05 which defined as statistically significant. When the experimental results showed significant heterogeneity $(P<0.05)$, random effect model is used, whereas the fixed effect model is used. If $\mathrm{I}^{2}$ index was less than/equal to $50 \%$, it is considered that there is no obvious heterogeneity among the results of these studies, and the fixed effect model is used for comprehensive analysis and processing of the data. The $95 \% \mathrm{CI}$ and the probability value are calculated. If $\mathrm{I}^{2}$ index more than/equal to $50 \%$, there is obvious heterogeneity among the various studies. The data included are analyzed synthetically and the random effect model is selected. Finally, the potential publication bias is analyzed by funnel plot.

\section{Result}

\subsection{Literature Screening Process}

Search CNKI database, WANFANG and other data published on the treatment of acute pancreatitis with integrated Chinese and Western medicine articles. Chinese search keywords are "Qingyi decoction; combined therapy of Chinese and Western medicine; Acute pancreatitis; randomized controlled; Meta analysis". Two reviewers independently screened the titles and abstracts of identified papers, and full text copies of all potentially relevant studies were obtained.

\subsection{Literature Screening Results}

According to the above retrieval methods, 200 related documents were preliminarily retrieved. After careful screening, a total of 27 articles met the above screening criteria, all of which were in Chinese, with a total of 2565 patients. The specific documents are listed in the table below (Table 1, Figure 1).

\subsection{Comparison Traditional Chinese and Western Medicine Treatment of Acute Pancreatitis with Western Medicine Alone}

A total of 27 articles were included, the total number of intervention group was 1342 cases, and the control group was 1223 cases. All 27 literatures were compared with those of Western medicine in the treatment of acute pancreatitis. From this result, we can see that the OR value of combined effect quantity is 4.98 , OR value $95 \% \mathrm{CI}$ is $3.79-6.54$. This interval does not include one, it can be considered that the OR value of combined effect quantity is not equal to one. 
Table 1. Characteristics of included studies and quality assessment.

\begin{tabular}{|c|c|c|c|c|c|}
\hline \multirow[t]{2}{*}{ Reference } & \multirow[t]{2}{*}{ Time } & \multicolumn{2}{|c|}{$\begin{array}{c}\text { Number of cases } \\
\text { (Comprehensive group) }\end{array}$} & \multicolumn{2}{|c|}{$\begin{array}{l}\text { Number of cases } \\
\text { (Single group) }\end{array}$} \\
\hline & & Effective & Total & Effective & Total \\
\hline Xinfeng Lin. et al. [10] & 2001 & 66 & 68 & 28 & 32 \\
\hline Changlin Lei. et al. [11] & 2007 & 31 & 34 & 21 & 34 \\
\hline Fengli Tian. et al. [12] & 2011 & 30 & 31 & 22 & 31 \\
\hline Wenwei Xu. et al. [13] & 2016 & 60 & 63 & 50 & 63 \\
\hline Pengcheng Liu. et al. [14] & 2013 & 72 & 75 & 47 & 71 \\
\hline Jing Zhang. et al. [15] & 2016 & 37 & 40 & 32 & 40 \\
\hline Jing Jiang. et al. [16] & 2009 & 68 & 70 & 44 & 50 \\
\hline Meng Cao. et al. [17] & 2010 & 32 & 34 & 25 & 31 \\
\hline Jidong Huang. et al. [18] & 2007 & 35 & 36 & 25 & 30 \\
\hline Moutong Liu. et al. [19] & 2015 & 83 & 85 & 72 & 85 \\
\hline Zerong Long. et al. [20] & 2003 & 34 & 36 & 25 & 30 \\
\hline Juan Fang. et al. [21] & 2009 & 38 & 40 & 32 & 40 \\
\hline Wenqiang Huang. et al. [22] & 2001 & 41 & 42 & 34 & 40 \\
\hline Shujin Qiu. et al. [23] & 2008 & 47 & 60 & 12 & 30 \\
\hline Qiongna Xing. et al. [24] & 2013 & 51 & 54 & 49 & 54 \\
\hline Wenying Dai. et al. [25] & 2014 & 29 & 30 & 26 & 30 \\
\hline Shujing He. et al. [26] & 2011 & 40 & 45 & 32 & 45 \\
\hline Hongliu Chen. et al. [27] & 2014 & 41 & 45 & 35 & 45 \\
\hline Peng Zhang. et al. [28] & 2010 & 34 & 40 & 27 & 40 \\
\hline Quan Hu. et al. [29] & 2011 & 35 & 36 & 32 & 36 \\
\hline Ke Wang. et al. [30] & 2010 & 44 & 46 & 35 & 40 \\
\hline Shicong Wang. et al. [31] & 2016 & 48 & 50 & 36 & 48 \\
\hline Yunfei Tu. et al. [32] & 2010 & 33 & 33 & 30 & 33 \\
\hline Juan Chen. et al. [33] & 2012 & 75 & 83 & 53 & 81 \\
\hline Jincheng Zhang. et al. [34] & 2009 & 48 & 50 & 40 & 48 \\
\hline Xainqiang Qi. et al. [35] & 2009 & 30 & 32 & 24 & 32 \\
\hline Jianghua Qu. et al. [36] & 2008 & 80 & 84 & 61 & 84 \\
\hline
\end{tabular}

The second row of the inverse of the text, the chi square value is $9.67, P=1.00>$ $0.05, \mathrm{I}^{2}=0 \%$. That shows the heterogeneity is low and the combined effect of the amount of OR value should be calculated by using a fixed effects model. That is: the effective rate of both methods is not the same. There is a statistical difference. The whole confidence interval is greater than 1 , it shows that the effective rate of treatment of acute pancreatitis with integrated traditional Chinese and western medicine is higher than that of patients treated by western medicine alone. In other words, the effect of integrated traditional Chinese and western medicine on acute pancreatitis is better than that of western medicine alone in the treatment of acute pancreatitis (Figure 2). Funnel plot can be seen on both 


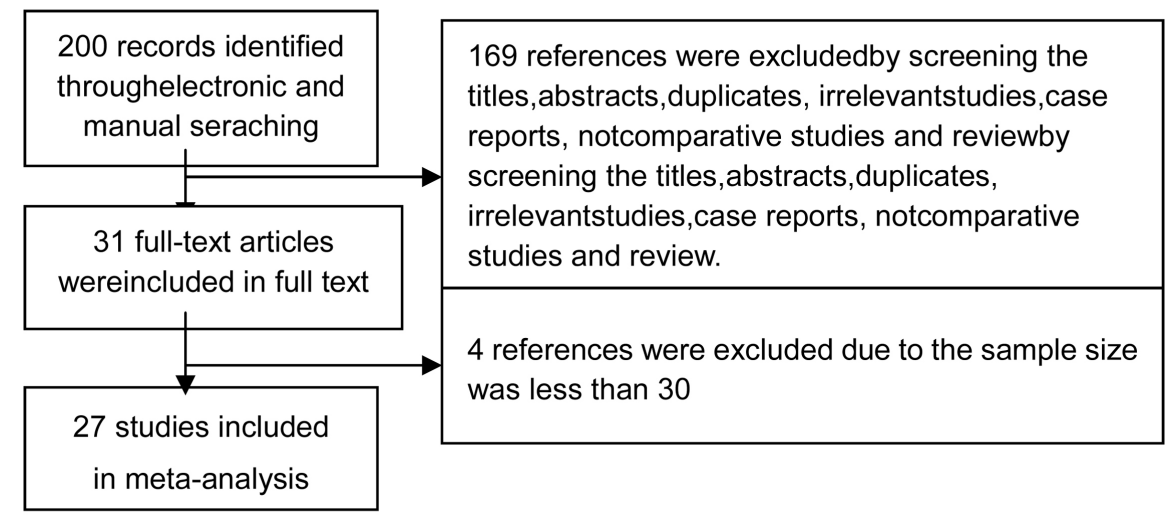

Figure 1. The flow chart shows the article selection process we performed.

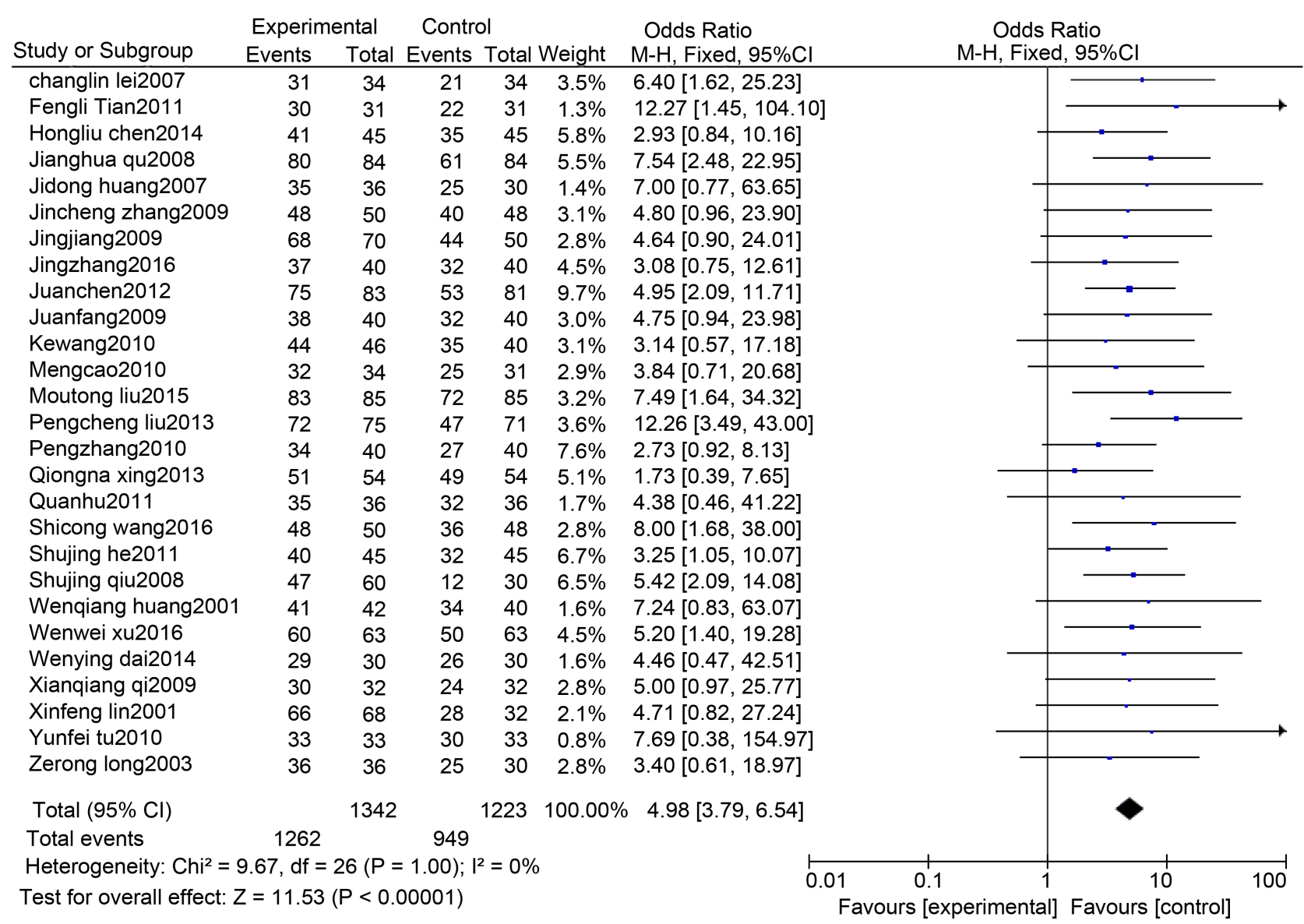

Figure 2. Forest plot: Comparison between traditional Chinese and Western medicine treatment of acute pancreatitis and that of western medicine alone.

sides of the more symmetrical, the publication bias is small and the data is more stable (Figure 3).

\section{Discussions}

Chinese medicine is the gem of ancient Chinese science, and also a key to open the treasure of Chinese civilization. Nevertheless, with the introduction of western 


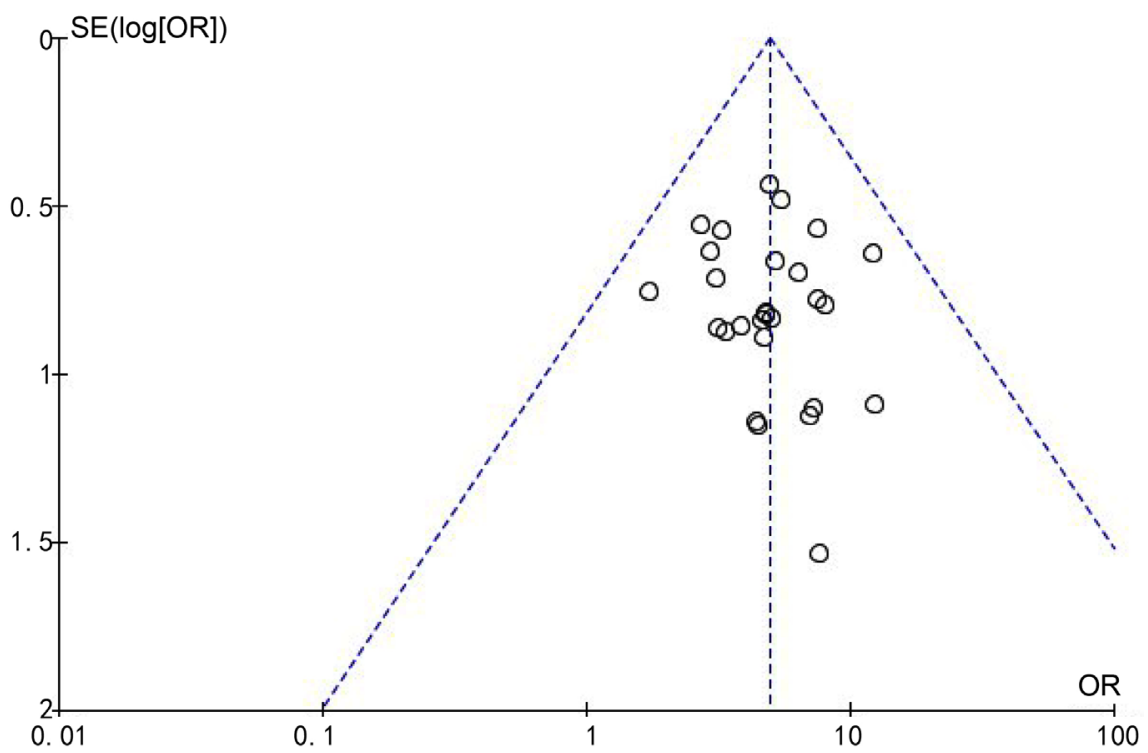

Figure 3. Funnel plot: Comparison between traditional Chinese and Western medicine treatment of acute pancreatitis and that of western medicine alone.

medicine and other reasons, Chinese medicine gradually declined. Interestingly, with the development of medicine, the application of scientific research methods, many traditional Chinese medicine treatment methods have been scientifically confirmed; traditional Chinese medicine is gradually paid attention to by medical researcher; traditional Chinese medicine and western medicine are no longer antagonistic and in isolated position; and the treatment of diseases with integrated traditional Chinese and western medicine is also the general trend. This article aims to further confirm the curative effect of integrated traditional Chinese and western medicine in the treatment of acute pancreatitis by statistical method.

Acute pancreatitis is an inflammatory reaction that activates pancreatic enzymes in the pancreas and digests the pancreatic tissue. These pathological changes were composed to tissue edema, bleeding and even necrosis. Clinical features of the disease were included to acute epigastric pain, nausea, vomit, fever, and elevated serum trypsin. The pancreas produces chemical inflammation due to its own digestion, involving inflammatory mediators and cytokines, which in turn leads to pancreatic microcirculation and intestinal failure, intestinal mucosal barrier change, intestinal microflora translocation, and so on. Then, the disease was further aggravated, and traditional Chinese medicine preparations such as Qingyi decoction had the effects of soothing the liver and regulating qi, clearing heat and relieving defecation, restoring the function of the depleted intestine, promoting the elimination of toxins and inflammatory factors, improving the intestinal microcirculation, and restoring the mucosal barrier function of the intestine. Chengtang $\mathrm{Wu}$ [1] found that Qingyi decoction could significantly reduce the pathological damage of pancreas and intestinal tissue of dogs. It also significantly inhibited the proliferation of Escherichia coli 
and Bacterioid bacteria, protected Bifidobacterium and Lactobacillus, increased intestinal colonization resistance, and significantly reduced plasma endotoxin level. Next, Muxin Wei [2] also found that Qingyi decoction not only could increase the level of serum motilin and decrease the level of serum amylase in rats, alleviate the pathological damage of pancreatitis, and protect the intestinal mucosal barrier, but also significantly improve gastrointestinal motility, achieve the excretion of toxins, adjust the intestinal microecological balance, and reduce the damage of endotoxin and various cytokines. Li Min [3] found that Qingyi decoction has antagonistic effect on acute pancreatitis induced by Rana frog peptide and lipopolysaccharide in mice by mouse model, which may be related to the clearance of intestinal bacteria. All of these were consistent with the concept of treatment of TCM-Liufu Yitong and the effects of Qingyi decoction on promoting gastrointestinal motility. Based on the conclusion of animal modeling experiment, many of clinicians such as Xiaoyun Jiang [4] and Kechen [5] carried out randomized controlled experiments and found that the effect of adding Qingyi decoction on the basis of routine western medicine treatment was better than that of western medicine alone in treating acute pancreatitis. Qingyi decoction has certain effect on the prevention of severe pancreatitis complicated with lung injury [6] and Post-ERCP pancreatitis [7]. But the single experimental sample size is often small, in order to avoid the limitation of single small sample conclusion, Pengyu [8] and Leili [9] made a systematic evaluation on the treatment of severe pancreatitis with Qingyi decoction. It is further proved that Qingyi decoction can significantly reduce the mortality rate of severe acute pancreatitis. The purpose of this study was to evaluate the efficacy of Qingyi decoction combined with conventional western medicine in the treatment of acute pancreatitis.

The treatment of acute pancreatitis with integrated traditional Chinese and western medicine plays a complementary role in clinical practice, which changes from early diagnosed and immediately surgery, conservative internal medicine and Individualized treatment, to the present combination of traditional Chinese and Western medicine in the treatment of acute pancreatitis. The clinical treatment point of view is also gradually changed under the guidance of scientific research. In this paper, we search for the relevant qualified documents through the databases of China knowledge Network, WANFANG and so on, and compare the therapeutic effects of integrated traditional Chinese and western medicine with those of simple western medicine in the treatment by means of meta analysis. Statistical data show that the treatment of acute pancreatitis by integrated traditional Chinese and western medicine is superior to that of western medicine alone, which is consistent with the current clinical research, and provides the corresponding evidence-based basis for clinical work and builds a bridge between traditional Chinese and western medicine. However, the deficiency of this paper lies in the lack of support from English literature, which is related to the fact that traditional Chinese medicine is a traditional medicine in China and the 
western experiments are relatively few. Despite the above limitations, our study is still deserved to be believed. with the application of scientific research methods and evidence-based evidence in the field of traditional Chinese medicine, more traditional Chinese medicine preparations and integrated Chinese and western medicine treatment will be gradually recognized by the international, more and more diseases will obtain better curative effect due to the combination of Chinese and Western medicine.

\section{References}

[1] Wu, C.T., Li, Z.L., Huang, X.C., et al. (1999) Comparative Study on the Effects of Qingwei Decoction and Bifidobacterium Mixture on the Intestinal Bacterial Translocation of Acute Necrotizing Pancreatitis. World Chinese Digest Journal, No. 6, 69-72.

[2] Wei, M.X., Li, J.J., Wang, X.X., et al. (2004) Mechanism of Enterodynamic Action in the Treatment of Acute Pancreatitis Rats in the Treatment of Pancreatic Decoction. World Chinese Digest Journal, 12, 1369-1372. https://doi.org/10.11569/wcjd.v12.i6.1369

[3] Li, M., Lin, S.S., Shen, L., et al. (2015) Pancreatic Qing Soup II Number Granule on the Protective Effect and Mechanism of Acute Pancreatitis in Mice. Chinese Journal of Pathology, Physiology, No. 7, 1282-1287.

[4] Jiang, X.Y., Gu, Y., Wang, Y.S., et al. (2009) Observation on the Treatment of Acute Pancreatitis Treated with Clear Pancreatitis. Hainan Medicine, No. 3, 32-33.

[5] Chen, K., Xu, T. and Mei, S.T. (2016) Clinical Efficacy and Safety of Nasal Jejunum Injection in Patients with Acute Severe Pancreatitis. Chinese Journal of Emergency Medicine, 25, 1439-1442.

[6] Ye, G.F. (2016) The Study on the Efficacy of the Combined Treatment of Acute Pancreatitis in Patients with Severe Acute Pancreatitis. Chinese General Medicine, No. 5, 872-874.

[7] Liu, C.G., Leng, D.Y., Liu, H., et al. (2009) Clinical Study on the Prevention of Pancreatitis and High Amylase after Endoscopic Retrograde Pancreaticobiliary Pancreatitis. Chinese Journal of Traditional Chinese and Western Medicine, No. 7, 607-610.

[8] Yu, P., Zeng, Q.Q., Wang, H.C., et al. (2008) Systematic Evaluation of the Treatment Value of Severe Acute Pancreatitis by Qingwei Decoction. Journal of Practical Medicine, No. 4, 544-547.

[9] Li, L., Liu, J. and Qin, K. (2015) A Meta-Analysis of the Clinical Efficacy of Qingwei Decoction in the Treatment of Severe Acute Pancreatitis. Journal of Chinese Experimental Formulation, No. 23, 207-211.

[10] Lin, X.F., Liu, N. and Zhu, M. (2001) Treatment of Acute Pancreatitis in Combination with Traditional Chinese and Western Medicine. Chinese Medicine Emergency, 10, 329-330.

[11] Lei, C.L. (2007) Observation on the Curative Effect of Chinese and Western Medicine on Acute Pancreatitis. Hubei Journal of Traditional Chinese Medicine, 29, 24.

[12] Tian, F.L., Hao, X., Gao, J.M., et al. (2011) Treatment of 62 Cases of Acute Pancreatitis Treated by Chinese and Western Medicine. Hebei Medicine, 33, 1083-1084.

[13] Xu, W.W., Shi, L. and Wei, W. (2016) The Curative Effect of Chinese and Western Medicine in Treating 126 Cases of Acute Pancreatitis. Zhejiang Clinical Medicine, 
18, $1127-1128$.

[14] Liu, P.C. (2013) Treatment of 146 Cases of Acute Pancreatitis Treated by Chinese and Western Medicine. Chinese Medical Emergency, 22, 308-310.

[15] Zhang, J. (2016) Observation on the Curative Effect of Chinese and Western Medicine on Acute Pancreatitis. Traditional Medicine in Asia Pacific, 12, 124-125.

[16] Jiang, J. (2009) Observation on the Curative Effect of Traditional Chinese and Western Medicine on Acute Pancreatitis. Chinese Primary Medicine, 16, 912-913.

[17] Cao, M. (2010) Observation on the Curative Effect of Traditional Chinese and Western Medicine on Acute Pancreatitis. Medical Review, 16, 1112-1113.

[18] Huang, J.D. and Chen, G.S. (2007) Treatment of 36 Cases of Acute Pancreatitis Treated by Chinese and Western Medicine. New Chinese Medicine, 39, 55-56.

[19] Liu, M.T. (2015) Observation on the Curative Effect of Traditional Chinese and Western Medicine on Acute Pancreatitis. Journal of Cardiovascular Diseases, Chinese and Western Medicine, 3, 23-25.

[20] Long, Z.R., Liu, L.C., Wang, X., et al. (2003) Treatment of Acute Pancreatitis Treated by Chinese and Western Medicine. Journal of Chinese Medicine, 31, 56-57.

[21] Fang, J., Li, X.X., Ou, J.-A., et al. (2009) Observation and Nursing of Curative Effect of Chinese and Western Medicine on Acute Pancreatitis. Chinese Medicine Guide, 7, 28-29.

[22] Huang, W.Q., Zhong, M.W., Wu, Y.Q., et al. (2001) Treatment of Acute Pancreatitis by Chinese and Western Medicine. New Chinese Medicine, 33, 27-28.

[23] Qiu, S.J. (2008) The Curative Effect of Traditional Chinese and Western Medicine on Severe Acute Pancreatitis. Chinese Medicine Emergency, 17, 614-621.

[24] Xing, Q.N. (2013) Observation on Clinical Efficacy of Chinese and Western Medicine in Treating Acute Pancreatitis. Traditional Medicine in Asia Pacific, 9, 76-77.

[25] Dai, W.Y., Liang, J.M., Xu, M.K., et al. (2014) Treatment of 60 Cases of Light Acute Pancreatitis Treated by Chinese and Western Medicine. Modern Clinical Medicine, 40, 124-125.

[26] He, S.J. (2011) Clinical Observation of 45 Cases of Acute Pancreatitis Treated by Chinese and Western Medicine. Clinical Medical Engineering, 18, 257-258.

[27] Chen, H.L. and Jiang, J.L. (2014) Observation on the Curative Effect of Chinese and Western Medicine on Acute Pancreatitis. Journal of Hubei University of Nationalities (Medical Edition), 31, 66-67, 69.

[28] Zhang, P. (2010) Clinical Observation of 40 Cases of Acute Pancreatitis Treated by Chinese and Western Medicine. Journal of Practical Chinese Medicine, 24, 76-77.

[29] Hu, Q. (2011) Observation on the Curative Effect of Traditional Chinese and Western Medicine on Acute Pancreatitis. Clinical Research of Chinese Medicine, 3, 24-25.

[30] Wang, K. and Zhou, Z.L. (2010) Observation on the Curative Effect of Chinese and Western Medicine on Acute Pancreatitis. Traditional Medicine in Asia Pacific, 6, 45-46.

[31] Wang, S.C. (2016) Observation on Curative Effect of Combined Chinese and Western Medicine for Acute Pancreatitis. Health Care Guide for Health Care, 48, 130.

[32] Tu, Y.F., Zheng, L. and Chen, Y.S. (2010) Treatment of 33 Cases of Acute Pancreatitis Treated by Chinese and Western Medicine. National Hospital BBS, 25, 31-32.

[33] Chen, J. (2014) Treatment of 80 Cases of Severe Acute Pancreatitis Treated by Chinese and Western Medicine. Chinese Journal of Medicine, 14, 57, 87. 
[34] Zhang, J.C. and Zheng, J. (2009) Observation on the Curative Effect of Traditional Chinese and Western Medicine in Acute Pancreatitis. Journal of Xinjiang Medical University, 32, 489-490.

[35] Qi, X.Q. (2009) The Results of Treatment of 32 Cases of Acute Pancreatitis Treated by Chinese and Western Medicine. Hunan Journal of Traditional Chinese Medicine, 25, 24-25.

[36] Qu, J.H. (2008) Clinical Observation of 84 Cases of Acute Pancreatitis Treated by Chinese and Western Medicine. Modern Clinical Medicine, 34, 284-285. 